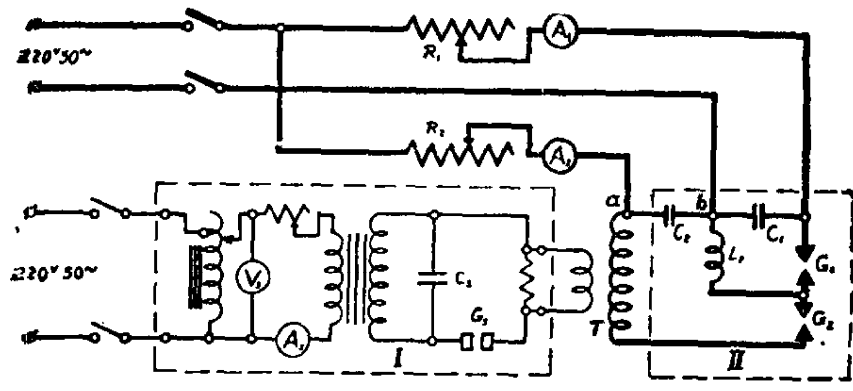

圆 1 双论础基本纨路简圆

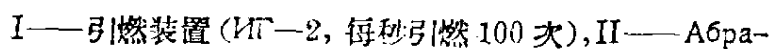
мсон 附加中路, T--Tesla 变压器, $C_{1}-L_{1}-G_{1}-$ 激發放电的论容放电迥路, $\mathrm{G}_{1}$ 一激發电讪, $\mathrm{G}_{2}$ 一加 热允弧， $\mathrm{C}_{2}$ 一一隔淔电谷。粗綫表示双近弧强流部分

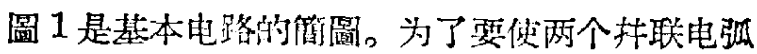
能同时引燃，我們乐用了 Абрамсон[6]的"付加电路，抹 加以修改, 使它合乎我們的裂求。乎們目前使用 $U \Gamma-2$ 型电花發生器作为设燃装置, 显然, 利用其他类型的

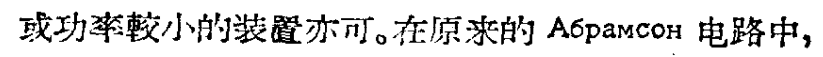
$c_{2}$ 只是一个輔助电花隲, 現在在 $a 、 b$ 两点間接入低 EE电隐, 抖去掉一个隔㨁电容, $G_{2}$ 也就成为一个功 焏可达几百正特以上的加热电弧了。除此之外, 电路 虳宁燃作用原理和 Абрамсон 所迅的一样。

改变电路中的 $R_{2}$ 則可改变加热电弧的电流, 而 激發条件不变。改变 $R_{1} 、 C_{1} 、 L_{1}$ 及 $G_{1}$ 則可改变激發 绦件，而不影响加热电㼋。

目的这电路还有这梢的缺点，即在某些条件下

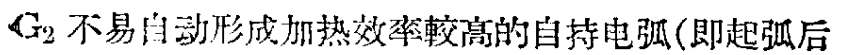
不必再引㦓也能維持不灭的电弧)。但是只要在通电后 将 $G_{2}$ 的玩个石丝电整互碰一下，自持电弧就形成了。

践们曾以含 $\mathrm{ZnO}$ 及 $\mathrm{CdO}$ 各 $0.1 \%$ 的 $\mathrm{SiO}_{2}$ 为对 潒，以数利条件进行多次摄㬐，以試驗这光源的再現 性。所用的电極㧧統如 圈2所示, 加热电流用16 安培，激登放电用普通 引燃交流电弧以及 $\mathrm{L}_{1}$ 的 为 $0.5 \mathrm{mH}_{1} \mathrm{C}_{1}$ 为 20 及 . $50 \mu \mathrm{F}$ 的电容放电。用 这些条件都得到士12\% 公右的均方偏美（所用 和为 $\mathrm{Zn} \mathrm{3392.59/Cd}$ :3261.06)。应部指出，这 只是初步試駿的結果， 所用的工作条件不能馀 是最好的; 扭且所用的

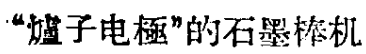
㭜性能很茬，粘构很不 均匀。这些都是能㨁接 影响再現性的因素。但

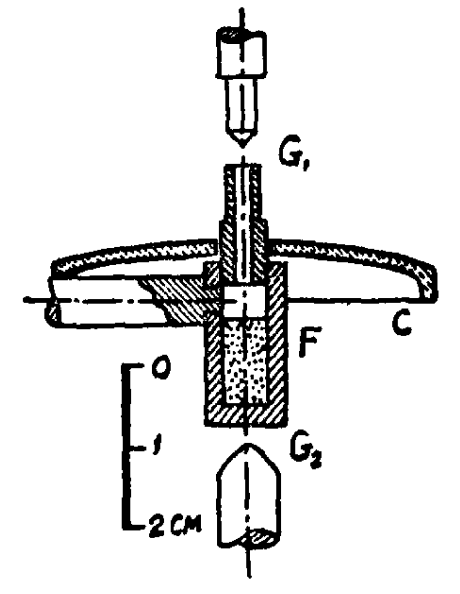

圖 2 代欇柔絖示意圆 $\mathrm{G}_{1}$ 一激發比䋇， $\mathrm{G}_{2}$ 一加热 电弧，F一一装有試样的“㯰子

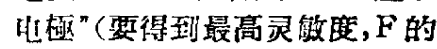
尺寸可以适当地增大, 以装盛较 多留試样)，C一坩佩盖，頂上 銘有小孔。
是，尽管如此，我倐所得到的再現性已綛比前迅文嗝 所报导的都好。

此外，我們还初步进行了一些利用不同的激策

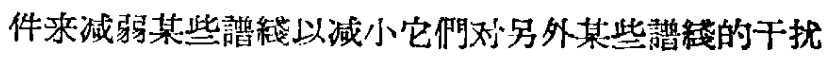
的試驗，这些試驗的結果都是很有希㕵的。

如果姴得到更高的加热效案，可用直流电作为电 路中浊流部分的电源，抹以“嘘子电極”为陽極。这时 在 $\mathrm{C}_{1}$ 的充电迴路中須加入一个较大的鉄心据流圈, 怙且 $R_{1}$ 不能太小。

目前秘們正对这种电路作进一步的的究和改进, 抹作备用它来进行某些非金屬元采分析的研究。

作者对这用化学研究所哭学周所长对本工作所給了的关

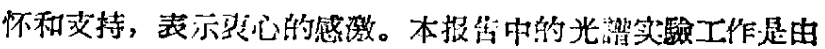

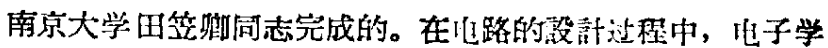
研究肵楼弊明同志提供了不少有益的意見。作者对田、楼两 同志表示感謝。

\section{黃 本立}

(中国科学院应用化学磔究所) 1957 年 4 月 19 日

[1] D. M. Shaw, O. I. Joensuu and L. H. Ahrens, Spectrochimica Acta, 4, 233(1950).

[2] D. M. Shaw, Geochim. et Cosmochim. Acta, 2, 118(1952).

[3] D. M. Shaw, 同.上, 2,185(1952).

[4] K. H. Wedepohl, 同. E, 3,93(1953).

[5] М. Л. Шиллинг, Зс.вод. Лсб., 22,447(1950).

[6] С. И. Абрамсон, 同. L., 14,1135(1948).

\section{二亚硒酸三軍鈵, $\mathrm{NaH}_{3}\left(\mathrm{SeO}_{3}\right)_{2}$ 的晶体結构}

\section{二亚硒酸三昰釣昆体乐掞 J. Janitzki 的工作[1]} 制得，其含硒量的实驗値和計算值各为 56.51 和 $56.42 \%$ 。晶体的 Laue 对称性为 $C_{2 h}$ 。晶体单科晶胞 的参数为

$$
\begin{aligned}
& a=5.90 \pm 0.02 \dot{A} \\
& b=4.82 \pm 0.02 \AA \\
& c=10.19 \pm 0.03 \dot{A} \\
& \beta=91^{\circ} 20^{\prime}
\end{aligned}
$$

晶胞中会有 $2\left\{\mathrm{NaH}_{3}\left(\mathrm{SeO}_{3}\right)_{2}\right\}$ 。泌度的許算值和实驗 值各为 $3.22_{4}$ 和 $3.21 \pm 0.01$ 克/ 暒米3。根据系統消光, 昆体結构中存在着滑移面 $n$ 。最后根据全部原子的荟 数值, 晶体的空間群确定为 $C_{s}^{2}-P n_{0}$ 。

根据 Weissenberg 圖 hol 上各行射点的分布情 况, 可为結构在 $(010)$ 面上的投影引㑁一 $-a^{\prime}=5.94 \AA$, $b^{\prime}=5.90 \AA, \gamma^{\prime}=62^{\circ}$ 的假六方单位, 后孝的假平面群 
为 $\mathrm{p} 6 \mathrm{~m}$ 。假六方单位的 $\underline{a}^{\prime}=1 / 2(\underline{c}-\underline{a}), \underline{b^{\prime}}=\underline{a}$ 。依据 这个䋊索, Patterson 投影 $\mathrm{P}(\mathrm{uw})$ 給出了 $\mathrm{Se}$ 和 $\mathrm{Na}$ 原子的 $x, z$ 坐标, 而 $\mathrm{P}(\mathrm{vw})$ 給出了它們的 $y$ 坐标。 从这些秲数看来，晶体中的 $\mathrm{Se}$ 和 $\mathrm{Na}$ 原子系按空間群 $C_{2 h}^{5}-P \frac{2_{1}}{n}$ 分布。依靠上迅重原子的参数找按空間群 $C_{2 h}^{5}$ 計算了电子密度分布函数 $\rho(x, z)$ 和 $\rho(y, z)$ 。計 算絬果指出, 晶胞中的氧原子系按空間群 $C_{s}^{2}$ 的对称 性分布。最后按空間群 $C_{s}^{2}$ 对上迅电子密度分布函数 逐步进行修正，取得了圖 1 和圖 2 中的結果。現将各 原子的参数列于表 1。晶体的䊅构示出于圖 3 中。从

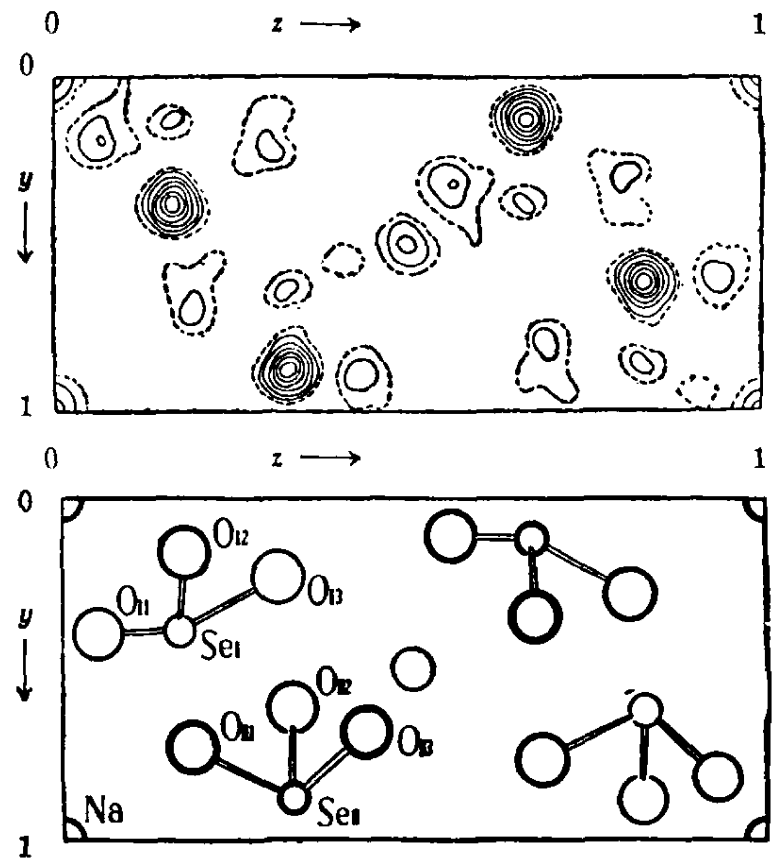

回 1 电子密度分布函数 $O(x, z)$ 及晶体結构在 $(010)$ 平面、上的投影

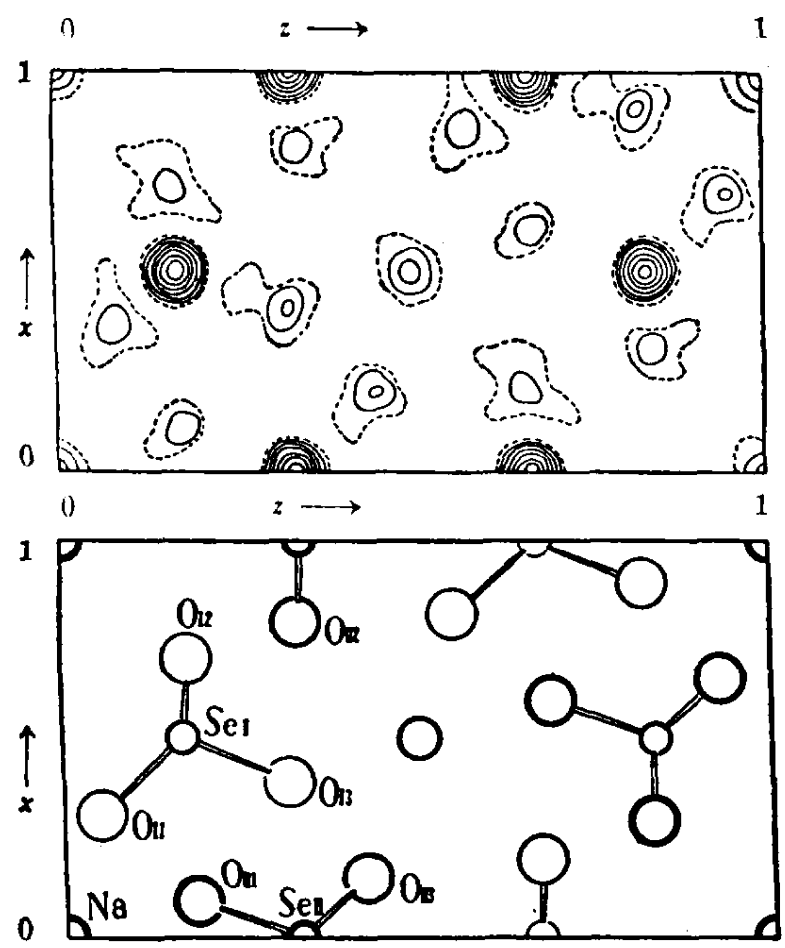

圆 2 电子密度分布函数 $O(y, z)$ 及晶体䊀构在 $(100)$ 平面上的投影
表 1

\begin{tabular}{llll}
\hline & $x$ & $y$ & $z$ \\
\hline $\mathrm{Na}$ & 0 & 0 & 0 \\
$\mathrm{Se}_{\mathrm{I}}$ & 0.500 & 0.336 & 0.169 \\
$\mathrm{Se}_{\mathrm{II}}$ & 0.000 & 0.884 & 0.331 \\
$\mathrm{O}_{11}$ & 0.340 & 0.192 & 0.067 \\
$\mathrm{O}_{12}$ & 0.733 & 0.145 & 0.170 \\
$\mathrm{O}_{13}$ & 0.400 & 0.249 & 0.313 \\
$\mathrm{O}_{111}$ & 0.125 & 0.734 & 0.184 \\
$\mathrm{O}_{112}$ & 0.795 & 0.667 & 0.331 \\
$\mathrm{O}_{113}$ & 0.200 & 0.887 & 0.448 \\
\hline
\end{tabular}

上述結构数据可見, 晶体系由离子 $\mathrm{Na}^{+}, \mathrm{HSeO}_{3}-$ 和分子 $\mathrm{H}_{2} \mathrm{SeO}_{3}$ 組成, 其中分子 $\mathrm{H}_{2} \mathrm{SeO}_{3}$ 和离子

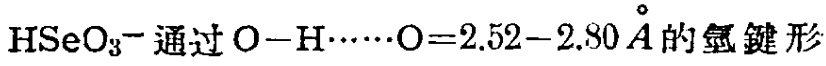
成圖 4 中的鍕型氯鍵体系，离子 $\mathrm{Na}^{+}$则处在一峝 6

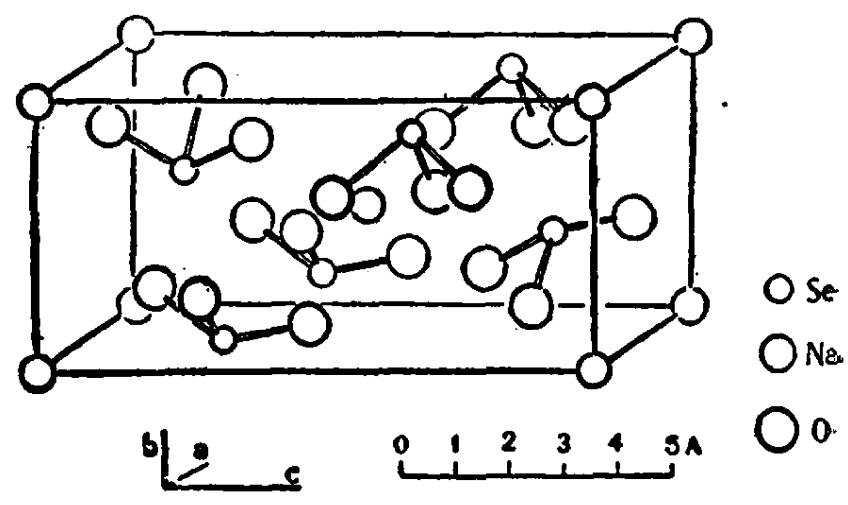

圆 3 晶体的結构

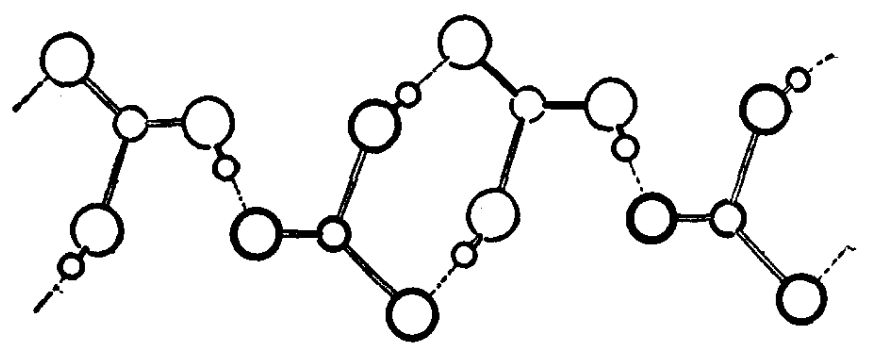

国 4 晶体中分子 $\mathrm{H}_{2} \mathrm{SeO}_{3}$ 和离子 $\mathrm{HSeO}_{3}-$ 形成的鐩型悬鍵体系

个O 原子按 $\mathrm{Na}-\mathrm{O}=2.32-2.58 \AA$ 形成的八面体配 位中。晶体中分子 $\mathrm{H}_{2} \mathrm{SeO}_{3}$ 和离子 $\mathrm{HSeO}_{3}$ - 的构型 各相当于結构式

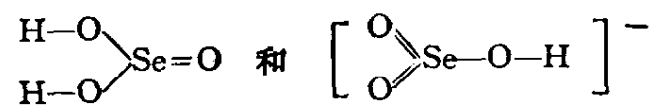

分子 $\mathrm{H}_{2} \mathrm{SeO}_{3}$ 的构型与亚硒酸晶体中婪[2]杆無二玫， 而亚硒酸亜根离子的构型份系首次测定。其他亚酩酸 盐和有关的亚硫酸盐的工作正在进行中。

周公度唐有褀

(北淙大学化学系, 中国科学院应用物理研究所) 1957 年 4 月 18 日

[ 1 ] J. Janitzki, Z. Anorg. allgem. Chem.,205, 49(1932)

[ 2 ] A. F. Wells, J. Chem. Soc., 1282(1949). 